\title{
Influence of foam rolling on the functional limitations of the musculoskeletal system in healthy women
}

\author{
Dariusz Boguszewski ${ }^{1}$, Magdalena Falkowska ${ }^{1}$, Jakub Grzegorz Adamczyk ${ }^{1,2}$, Dariusz \\ Białoszewski ${ }^{1}$
}

${ }^{1}$ Department of Rehabilitation, Physiotherapy Division. Medical University of Warsaw, Poland; ${ }^{2}$ Department of Theory of Sport, Józef Piłsudski Academy of Physical Education, Warsaw, Poland

\section{Summary}

Study aim: To determine the effect of foam rolling on the functional limitations of the musculoskeletal system.

Material and methods: The study encompassed 37 healthy and physically active women divided into two groups. Group 1 comprised women who performed self-massage with a foam roller after physical effort twice a week, for two months. Group 2 (control) comprised women who did not undergo any exercises or treatment after physical effort. The study used the following research tools: the Functional Movement Screen (FMS) test, the Core Muscle Strength and Stability Test (CMS\&ST), and the Sit and Reach Test.

Results: The study revealed that foam rolling minimized functional limitations (as measured with the FMS test). The differences between the first and second measurement in Group 1 were statistically significant $(p=0.014)$. In the control group, the results of both measurements were similar. In the CMS\&ST, the maximal result of three minutes was not achieved. Moreover, no improvement of results was observed. In the Sit and Reach Test, a statistically significant improvement in the flexibility of the posterior muscles of the thigh was noted in Group 1 (right lower limb $p=0.009$, left lower $\operatorname{limb} p=0.007$ ).

Conclusions: 1. Foam rolling may minimize the functional limitations of the musculoskeletal system. It is recommended to incorporate self-myofascial release techniques after physical effort into training. 2. Using foam rolling helped maintain the results obtained in the Core Muscle Strength and Stability Test. Therefore, foam rolling may help maintain the achieved core stability.

\section{Key words: Foam rolling - FMS - Range of motion - Self-massage - Myofascial relaxation}

\section{Introduction}

In view of the growing interest in physical activity and recreational participation in sports, it is important to promote safety of movement and prevent bodily injury [40]. The risk of sustaining a bodily injury can be assessed by using various methods, such as the Flamingo Test, Rotational Test, Test of Susceptibility to Injury During Falls, Movement Compensation Screen, Core Muscle Strength and Stability Test, Thomas Test, and Dega Test [9, 19, 20, 23, $31,32]$. The tests evaluate balance, range of motion in the joints, and the quality of motion, i.e., the reproduction of complex movement patterns; for there are various causes of bodily injuries. However, injuries most often result from the combined effect of microtraumas, excessive strain on the musculoskeletal system, or inappropriate warm-up [16].
A comprehensive functional evaluation of the musculoskeletal system in sports can also be conducted with the Functional Movement Screen (FMS) test. It is a screening test that allows for assessing the quality of motion, and motor asymmetries and dysfunctions, which can lead to injuries. Its usefulness has been proven in numerous scientific studies [1, 6, 13, 22, 24, 25, 26, 33]. Specifically, efficient functioning of kinematic chains in correct movement patterns and core stability make human motion safe, which decreases the risk of overstraining and potential tissue damage [3, 29].

Methods that help reduce the risk of bodily injury include introducing supplementary exercises into training. The exercises do not have a significant effect on achieved sports results, but they may make the muscles more flexible, and improve the ergonomics and symmetry of movement. This prepares the musculoskeletal system for greater 
strain during sports training $[5,35]$. Before an exercising program can be designed, the requirements posed by a given sports discipline should be analyzed. A functional approach to training allows for adopting forms of shaping movement that most closely replicate the conditions during competitions. The progression of motor tasks depends, to a large degree, on the level of fitness and abilities to perform a given exercise. This is why the evaluation of body stability and control, the aim of which is to identify specific functional deficits of the body, is an important consideration, as is the mobility of individual body segments and the entire kinematic chain, as well as muscle endurance and strength. The program should promote strengthening weakened structures and correcting faulty movement patterns [10].

Popular and effective exercises that supplement training include stabilizing exercises, as well as Pilates and stretching $[4,30,41]$. Moreover, physiotherapy treatments, such as various forms of massage, are becoming increasingly popular $[2,7,17,39]$. Among these is selfmassage with a foam roller, i.e., a self-myofascial release technique. This form was first used in 1987 by Sean Gallagher, an American physical therapist. Currently, it is a technique that is gaining popularity among both therapists and athletes. Foam rolling is considered a procedure that facilitates post-exertional restitution, which in turn has a positive effect on flexibility $[18,34,38]$. However, the number of publications on the empirical verification of the effectiveness of this method is still insufficient. This is why the aim of this study was to determine the effect of foam rolling on functional limitations of the musculoskeletal system.

\section{Material and methods}

Study participants comprised 37 healthy, physically active women (female students at the Medical University of Warsaw), aged between 19 and 26 years, who attended fitness classes (step and fat burning) at least twice a week. The participants were randomly divided into two groups. Group 1 (Foam rolling) comprised women who performed self-massage with a foam roller after physical effort twice a week, for two months. Group 2 (Control) comprised women who did not undergo any exercises or treatment after physical effort (Table 1). Persons with chronic illnesses or damage to the musculoskeletal system that could affect performance during the tests and exercises or massage were excluded from the study.

On the first day of the study, before starting the exercises, each woman underwent the Functional Movement Screen (FMS) test, the Core Muscle Strength and Stability Test (CMS\&ST) and the Sit and Reach Test (for the right and the left side) $[11,14,15,27]$. Subsequently, persons from Group 1 performed a massage with a foam roller after effort according to instructions for about 20 minutes, twice a week, for two months. The study participants were familiarized with the correct methodology of performing a massage with a foam roller. The participants were instructed to focus more on rolling the muscle groups that were sorer and to massage slowly. The following body parts were massaged, in the following order: anterior part of the thigh, posterior part of the thigh, posterior part of the lower leg, lateral side of the thigh, medial part of the thigh, back, and the posterior part of the arm and shoulder. Exercise 1: The body in a forearm plank, a foam roller below the thighs. Movement: Vertical pushing movements with the arms; the area from the patella to the anterior inferior iliac spine is massaged. Exercise 2: The thigh of the massaged limb is placed on a foam roller, the other limb bent at the knee joint and the hip joint and supported on the ground. The upper limbs supported on the ground. Movement: Vertical pushing movements with the upper limbs, while the lower limb is supported on the ground; the area from the popliteal fossa to the ischial tuberosity is massaged. Exercise 3: The lower leg of the massaged lower limb is placed on a foam roller, the other limb bent at the knee joint and hip joint, and supported on the ground. The upper limbs supported on the ground. Movement: Vertical pushing movements with the upper limbs, while the lower limb is supported on the ground; the area from the calcaneal tuber to the popliteal fossa is massaged. Exercise 4: The body in a sideways forearm plank, the lateral surface of the thigh supported on a foam roller. The other limb bent at the knee joint and the hip joint and supported frontally on the ground. Movement: Vertical pushing movements with the supported lower limb. Exercise 5: The body in a forearm plank, the massaged lower limb bent and abducted at the hip joint and placed on a foam roller. Movement: Sideways pushing movements with the arms; the medial area of the thigh is massaged. Exercise 6: Lying on the back on a transversely placed foam roller, the upper limbs crossed on the chest, and the lower limbs bent at the knee joints.

Table 1. Characteristics of examined women

\begin{tabular}{lccccc}
\hline Groups & Number of people [n] & Age [years] & Body mass [kg] & Body height [cm] & BMI \\
\hline Foam rolling & 19 & $22.8 \pm 2.3$ & $60.1 \pm 6.6$ & $167.4 \pm 5.9$ & $21.4 \pm 1.7$ \\
Control & 18 & $24.4 \pm 1.6$ & $60.5 \pm 6.0$ & $166.3 \pm 4.9$ & $21.9 \pm 1.7$ \\
\hline
\end{tabular}


Movement: Vertical pushing movements with the lower limbs performed by bending and unbending the knees; the area of the back is massaged. Exercise 7: The body in a heel sit, the trunk bent forward, a foam roller under the arms, the elbow joints bent. Movement: Vertical pushing movements performed by moving the buttocks away from the heels; the area from the olecranon to the armpit is massaged. Exercise 8: The body on all fours, the massaged upper limb resting on a foam roller placed under the arm. Movement: Pushing movements to the right and left performed by twisting the trunk; the area from the olecranon to the glenohumeral joint is massaged. These exercises are performed at moderate speed [18].

After two months, each of the groups was tested again, as on the first day. The Functional Movement Screen test, Core Muscle Strength and Stability Test, and Sit and Reach Test were conducted $[11,14,15,27]$. The tested female students were also asked to complete a short survey (comprising biometric data, information on bodily injuries and their potential treatment).

Gray Cook and Lee Burton's [14, 15] Functional Movement Screen (FMS) was designed in order to perform an objective analysis of human movement patterns in terms of functional capacity and to predict and prevent injuries in athletes. Thanks to a three-plane movement evaluation it is possible to reveal abnormalities in kinematic chains, as well as to perform a comprehensive assessment revealing asymmetry and significant functional limitations resulting from incorrect mobility and stability of the musculoskeletal system $[14,15]$. The FMS comprises seven exercises testing basic movement patterns: 1. Deep Squat, 2. Hurdle Step, 3. In-Line Lunge, 4. Shoulder Mobility, 5. Active Straight Leg Raise (ASLR), Trunk Stability Push-Up, and 7. Rotary Stability. Performance in each task is evaluated on a four-point scale, from 0 to 3 points ( 3 points are given for a correct movement pattern, 2 points are given for a compensated movement pattern, 1 point is given when a movement pattern is not performed, and 0 points are given when a movement pattern or a provocation test is accompanied by pain). In total, a tested person may score 21 points. The FMS examination is conducted before exercises, before warm-up. The evaluation is carried out in two plains: sagittal and coronal. A tested person performs a given motor task three times, and the tester assesses the best attempt. If there are doubts whether a pattern was performed correctly, a lower score is awarded. Each side of the body is evaluated separately $[14,15]$.

To assess and measure the strength and endurance of core stability muscles, the participants were subjected to the Core Muscle Strength and Stability Test (CMS\&ST). The test was repeated twice, before and after the exercises. It involves holding a forearm plank position for three minutes. During the test, the plane of the support is decreased by lifting the limbs off the ground [27].
The range of motion of the ischiocrural muscles was evaluated with the Sit and Reach Test for the right and the left side. The test was carried out twice, before and after the exercises. The distance from the end of the index finger to the line of the toes was measured with a ruler. The position adopted during the test is a pike sit, with the nontested limb bent and pulled towards the chest and supported by the ipsilateral upper limb. A tested woman was to reach the toes of the straightened lower limb with the other upper limb. Inability to touch the toes indicates limited mobility of the posterior muscle chain, which may lead to the occurrence of incorrect movement patterns [11].

The data were analyzed using standard methods of statistical analysis and arithmetic means, including standard deviations. The relationships between the variables were determined with the Pearson correlation. The significance of differences between the results of each group was evaluated with the Mann-Whitney $U$ test, and the differences between the right and the left side were determined with the Wilcoxon signed-rank test. The level of significance was set at $\alpha=0.05$. The calculations were conducted using MS Excel and Statistica 10 software.

\section{Results}

The research showed that foam rolling minimized the functional limitations of the musculoskeletal system measured with the Functional Movement Screen test. The differences between the first and the second measurement in Group 1 (Foam rolling) were statistically significant $(p=0.014)$. In the control group, the results of both measurements were similar (Table 2).

Group 1 (Foam rolling) showed a statistically significant improvement of results in two trials: Hurdle Step $(p<0.001)$ and Active Straight Leg Raise $(p=0.007)$. The best results (in both measurements) were achieved in the Shoulder Mobility trial, and the lowest results were achieved in the trial of the strength and stability of the shoulder girdle muscles and trunk muscles, i.e., in the push-up trial. In the Trunk Stability Push-Up trial, one person scored 0 points in both measurements. In Group 2 (Control), the study did not reveal a significant improvement in the results of the FMS test. In the first measurement, 0 points were noted twice in the Shoulder Mobility trial, 0 points were noted in both measurements in the Trunk Stability Push-Up trial, and 0 points were noted during the second measurement in the Deep Squat test. The best results were observed in the shoulder girdle mobility test, and the lowest results were observed (as in the experimental groups) in the two other exercises. The greatest differences between the experimental group and the control group occurred in three trials: Hurdle Step, Shoulder Mobility, and Active Straight Leg Raise (Table 2). 
Table 2. Results of FMS test

\begin{tabular}{|c|c|c|c|c|c|c|c|c|c|}
\hline Groups & Measurements & $\begin{array}{l}\text { Deep } \\
\text { squat }\end{array}$ & $\begin{array}{c}\text { Hurdle } \\
\text { step }\end{array}$ & $\begin{array}{l}\text { In-line } \\
\text { lunge }\end{array}$ & $\begin{array}{l}\text { Shoulder } \\
\text { mobility }\end{array}$ & $\begin{array}{c}\text { Active } \\
\text { straight leg } \\
\text { raise }\end{array}$ & $\begin{array}{l}\text { Trunk } \\
\text { stability } \\
\text { push-up }\end{array}$ & $\begin{array}{l}\text { Rotary } \\
\text { stability }\end{array}$ & Sum \\
\hline \multirow{3}{*}{$\begin{array}{l}\text { Foam } \\
\text { rolling }\end{array}$} & before & 1.79 & 2.16 & 2.42 & $2.95^{*}$ & 2.16 & 1.16 & 1.32 & 13.95 \\
\hline & after & 1.74 & $2.74 *$ & 2.47 & 3.00 & $2.74 *$ & 1.16 & 1.53 & $15.37 *$ \\
\hline & differences & ns & $\mathrm{p}<0.001$ & ns & ns & $\mathrm{p}<0.01$ & ns & $\mathrm{p}<0.05$ & $\mathrm{p}<0.01$ \\
\hline \multirow{3}{*}{ Control } & before & 1.83 & 2.17 & 2.39 & 2.44 & 2.28 & 1.17 & 1.39 & 13.67 \\
\hline & after & 1.89 & 2.17 & 2.33 & 2.83 & 2.17 & 1.22 & 1.33 & 13.94 \\
\hline & differences & ns & ns & ns & ns & ns & ns & ns & ns \\
\hline
\end{tabular}

$* \mathrm{p}<0.05-$ differences between groups

Table 3. Results of CMS\&ST and Sit\&Reach tests

\begin{tabular}{llccc}
\hline Groups & Measurements & CMS\&ST [s] & Sit\&Reach R [cm] & Sit\&Reach L [cm] \\
\hline \multirow{3}{*}{ Foam rolling } & before & 46.24 & 1.05 & 0.74 \\
& after & $49.21^{*}$ & $4.68^{*}$ & $4.38^{*}$ \\
& differences & $\mathrm{ns}$ & $\mathrm{p}<0.01$ & $\mathrm{p}<0.05$ \\
\hline \multirow{3}{*}{ Control } & before & 45.72 & 0.47 & 0.59 \\
& after & 41.72 & -0.75 & -0.83 \\
& differences & $\mathrm{ns}$ & $\mathrm{ns}$ & $\mathrm{ns}$ \\
\hline
\end{tabular}

$* \mathrm{p}<0.05-$ differences between groups

None of the tested women achieved a maximum score (180 s) in the Core Muscle Strength and Stability Test (CMS\&ST). The results of the first and the second measurement were similar in both groups. In the Sit and Reach Test, a statistically significant improvement of the flexibility of the posterior muscles of the thigh in the Foam rolling Group was observed (right lower limb $p=0.009$, left lower $\operatorname{limb} p=0.007$ ). Furthermore, a statistically significant decrease of the flexibility of the posterior muscles of the thigh was observed in the Control Group (right lower $\operatorname{limb} p=0.018$, left lower limb $p=0.001$ ) (Table 3).

A high negative correlation was observed between the number of bodily injuries and the overall result of the FMS test (measurement 1). In the second measurement, the correlation was not significant $(r=-0.123)$. The strongest correlation among all the exercises in the test was noted in the case of Deep Squat $(r=-0.334, p<0.05)$, Active Straight Leg Raise $(r=-0.299, p<0.05)$, and Shoulder Mobility $(r=-0.287, p<0.05)$. The total result of the first FMS test correlated negatively with the number of bodily injuries in all groups. The strongest correlation concerned Group 1 (Foam rolling): $r=-0.633$ and $p<0.01$ (Table 4).
Low correlations were observed between the results of the FMS test and age, body height, body mass, and BMI.

High positive correlations were observed between the majority of the results of the Functional Movement Screen test and the Core Muscle Strength and Stability Test. The highest level of significance concerned the Control Group. A correlation between the result of the FMS test and the flexibility of the posterior muscles of the thigh was noted in the first measurement in both groups, and only in Group 1 (Foam rolling) for the left side in the second measurement (Table 5).

Table 4. Correlations between overall results of FMS and number of injuries

\begin{tabular}{lcc}
\hline Groups & Before & After \\
\hline Foam rolling & $-0.633^{*}$ & -0.092 \\
Control & -0.294 & -0.352 \\
All & $-0.413^{*}$ & -0.27 \\
\hline
\end{tabular}

$* \mathrm{p}<0.05$ 
Table 5. Correlations between results of tests

\begin{tabular}{lccc}
\hline Measurement 1 & & & \\
\hline Groups & FMS vs. CMS\&ST & FMS vs. Sit\&Reach R & FMS vs. Sit\&Reach L \\
Foam rolling & 0.163 & $0.535^{*}$ & $0.581^{*}$ \\
Control & $0.725^{*}$ & $0.460^{*}$ & $0.505^{*}$ \\
All & $0.521^{*}$ & $0.483^{*}$ & $0.526^{*}$ \\
\hline Measurement 2 & & & \\
\hline Groups & FMS vs. CMS\&ST & FMS vs. Sit\&Reach R & FMS vs. Sit\&Reach L \\
Foam rolling & 0.385 & 0.404 & $0.615^{*}$ \\
Control & $0.797^{*}$ & 0.361 & 0.387 \\
All & $0.677^{*}$ & $0.463^{*}$ & $0.531^{*}$ \\
\hline
\end{tabular}

$* \mathrm{p}<0.05$

\section{Discussion}

This study showed that self-massage with a foam roller has a positive effect on minimizing functional limitations, which may lead to the occurrence of bodily injuries and pain. This study revealed a statistically significant improvement of performing movement patterns and an increase in muscle flexibility after a set of exercises with a foam roller. Škarabot et al. [36] also observed a positive result of a foam roller. After using the above-mentioned method (and stretching), an increase of the passive range of motion in the tarsal joint was observed in young swimmers. The methods had a similar effect on increasing mobility in the tarsal joint when applied both separately and in combination [36]. Stevens observed an increase of mobility and functional capability of athletes, as well as a reduction of the likelihood of sustaining bodily injuries after a massage with a foam roller. Foam rolling improved joint mobility and the functionality of the massaged muscles [37]. Performing supplementary exercises (self-massage) as an active form of regeneration has a considerable advantage over the passive form of post-exertional regeneration. It prolongs muscle hyperemia, which facilitates their regeneration [28].

In the prophylaxis of bodily injuries, it is important to indicate the likelihood of sustaining an injury and to identify existing dysfunctions of the musculoskeletal system. This is possible thanks to the Functional Movement Screen test. The test is used for evaluating movement patterns and functional fitness of athletes and physically active persons $[1,13,22,24,25,26]$. Chorba et al. [12] researched the effect of the compensation of movement patterns on the predispositions for injuries in young female athletes. The study showed a significant negative correlation between the result of the FMS test and the occurrence of bodily injuries [12]. Garrison et al. [16] and Kiesel et al. [21] reached similar conclusions. They also revealed that results equaling 14 points and less indicate a predisposition for sustaining injury in the future. The groups tested as part of this study obtained an average score below 14 points in the first measurement. After eight weeks of applying selfrelaxation techniques using a foam roller, the exercising persons obtained a result above 15 points, which indicates moderate susceptibility to bodily injuries. Therefore, it can be assumed that foam rolling contributed to minimizing the risk of sustaining injuries.

The present study revealed significant positive correlations between the results of the Functional Movement Screen test and the Core Muscle Strength and Stability Test; however, two months of training combined with selfmassage using a foam roller did not result in the improvement of the result of the CMS\&ST. Similar correlations were observed by using core stability training in female basketball players [8].

The FMS test is a tool that is used frequently and is widely discussed in the literature. It is used among athletes of many sports disciplines. It allows for a functional evaluation of movement patterns and the predisposition to bodily injuries due to the practice of sports disciplines. The FMS may be used for determining the likelihood of sustaining bodily injuries and identifying weak points in athletes' kinematic chains. It allows for focusing on weak points and improving the functional fitness of athletes, and, as a consequence, reducing the risk of suffering a bodily injury $[1,12,21,25]$.

In scientific publications, there is a visible need to conduct a greater number of studies that will allow for broadening the knowledge on the effect of self-myofascial release on the musculoskeletal system, and comparing it with other methods. Research should also take into account the effects of self-myofascial release achieved by 
those participants who already had experience with this technique as well as the effects on those who only learned to use it during the study.

Considering further research, it is advisable to investigate a larger group, more varied in terms of sex, age, health state, height and weight, physical activity performed or, for example, lifestyle. Moreover, foam rolling may be supplemented with further physiotherapeutic treatment or exercises, assessing their influence on the body, as well as comparing the effect of foam rolling with other forms of treatment.

\section{Conclusions}

1. Foam rolling may minimize functional limitations of the musculoskeletal system. It is recommended to incorporate self-myofascial release techniques after physical effort into training as a means of preventing bodily injuries.

2. The FMS test provides us with information about functional limitations of the musculoskeletal system and its dysfunctions that can lead to damage. It may be a diagnostic tool that can be used in the prophylaxis of bodily injuries.

3. Studies on prophylactic programs should be continued in different groups, especially among athletes, for whom the risk of bodily injuries is the highest.

\section{Conflict of interest: Authors state no conflict of interest.}

\section{References}

1. Adamczyk J.G., D. Boguszewski, D. Białoszewski (2015) Functional assessment of male track and field runners through Functional Movement Screen test. Med. Sport., 68(4): 563-575.

2. Adamczyk J.G., I. Krasowska, D. Boguszewski, P. Reaburn (2016) The use of thermal imaging to assess the effectiveness of ice massage and cold-water immersion as methods for supporting post-exercise recovery. J. Therm. Biol., 60: 20-25. DOI: 10.1016/j.jtherbio.2016.05.006.

3. Akuthota V., A. Ferreiro, T. Moore, M. Fredericson (2008) Core Stability Exercise Principles. Curr. Sport. Med. Rep., 7(1): 39-44. DOI: 10.1097/01. CSMR.0000308663.13278.69.

4. Augustyn G., A. Wodnicka, Z. Szyguła (2013) The influence of stretching on delayed onset muscle soreness. Medicina Sportiva Practica, 14(4): 97-99.

5. Bahr R., K. Thorborg, J. Ekstrand (2015) Evidence-based hamstring injury prevention is not adopted by the majority of Champions League or Norwegian Premier League football teams: the Nordic Hamstring survey. $\mathrm{Br}$. $J$.
Sport. Med., 2015 May 20, DOI:10.1136/bjsports-2015094826.

6. Bock C., M. Stierli, B. Hinton (2016) The Functional Movement Screen as a predictor of police recruit occupational task performance. J. Bodyw. Mov. Ther, 20: 310-315. DOI: 10.1186/s12891-016-1146-0.

7. Boguszewski D., E. Kwapisz (2010) Sports massage and local cryotherapy as a way to reduce negative effects of rapid weight loss among kickboxing contestants. Arch. Budo, 6(1): 45-49.

8. Boguszewski D., N. Mrozek, J.G. Adamczyk, D. Białoszewski (2015) The influence of core stability exercises on functional limitations in female basketball players. Polish J. Sport. Med., 31(4): 187-195. DOI: 10.5604/1232406X.1193626.

9. Boguszewski D., J.G. Adamczyk, M. Buda, M. Kłoda, D. Białoszewski (2016) The use of functional tests to assess risk of injuries in judokas. Arch. Budo Sci. Martial Art. Extreme Sport., 12: 57-62.

10. Boyle M. (2004) Functional training for sports. Human Kinetics; Champaign.

11. Buckup K., J. Buckup (2016) Clinical Tests for the Musculoskeletal System: Examinations - Signs - Phenomena. Thieme, New York.

12. Chorba R., D. Chorba, L. Bouillon (2010) Use of a Functional Movement Screening tool to determine injury risk in female collegiate athletes. N. Am. J. Sports Phys. Ther., 5(2): 47-54.

13. Clifton D., D. Grooms, J. Onate (2015) Overhead deep squat performance predicts functional movement screen score, Int. J. Sports Phys. Ther, 10(5): 622-627.

14. Cook G., L. Burton, B.J. Hoogenboom, M. Voight (2014) Functional Movement Screening: the use of fundamental movements as an assessments of function - part 1. Int. J. Sports Phys. Ther., 3: 396-409.

15. Cook G., L. Burton, B.J. Hoogenboom, M. Voight (2014) Functional Movement Screening: the use of fundamental movements as an assessments of function - part 2. Int. J. Sports Phys. Ther., 4: 549-563.

16. Garrison G., R. Westrick, M. Johnson (2015) Association between the Functional Movement Screen and injury development in college athletes. Int. J. Sports Phys. Ther, 10(1): 21-28.

17. Gill N.D., C.M. Beaven, C. Cook (2006) Effectiveness of post-match recovery strategies in rugby players. Br. J. Sport. Med., 40: 260-263. DOI: 10.1136/ bjsm.2005.022483.

18. Harley K., D. Hatfield, P. Blanpied, L. Dorfman, D. Riebe (2014) The effects myofascial release with foam rolling on performance. J. Strength Cond. Res., 28: 61-68. DOI: 10.1519/JSC.0b013e3182956569.

19. Kalina R.M., B. Barczyński, K. Klukowski, J. Langfort, B. Gassienica-Walczak (2011) The method to evaluate the susceptibility of injuries during the fall - validation 
procedure of the specific motor test. Arch. Budo, 7(4): 201-215.

20. Kalina R.M., W. Jagiełło, B.J. Barczyński (2013) The method to evaluate the body balance disturbation tolerance skills - validation procedure of the Rotational Test. Arch. Budo, 9(1): 59-80.

21. Kiesel K., P. Plisky, M. Voight (2007) Can serious injury in professional football be predicted by a preseason Functional Movement Screen? N. Am. J. Sports Phys. Ther, 2(3): 147-158.

22. Kodesh E., E. Shargal, R. Kislev-Cohen, S. Funk, L. Dorfman, G. Samuelly, J.R. Hoffman, N. Sharvit (2015) Examination of the Effectiveness of Predictors for Musculoskeletal Injuries in Female Soldiers. J. Sports Sci. Med., 14: 515-521.

23. Ladeira C.E., L. Hess, B. Galin, S. Fradera, M. Harkness (2005) Validation of an abdominal muscle strength test with dynamometry. J. Strength Cond. Res., 19(4): 925-930. DOI: 10.1519/R-16664.1.

24. Letafatkar A., M. Hadadnezhad, S. Shojaedin, E. Mohamadi (2014) Relationship between Functional Movement Screening score and history of injury. Int. J. Sports Phys. Ther, 1: 21-27.

25. Lockie R.G., A.B. Schultz, S.J. Callaghan (2015) A preliminary investigation into the relationship between functional movement screen scores and athletic physical performance in female team sport athlete. Biol. Sport, 32: 41-51. DOI: 10.5604/20831862.1127281.

26. Lockie R.G., A.B. Schultz, C.A. Jordan, S.J. Callaghan, M.D. Jeffriess, T.M. Luczo (2015) Can selected functional movement screen assessments be used to identify movement deficiencies that could affect multidirectional speed and jump performance? J. Strength Cond. Res., 29(1): 195-205. DOI: 10.1519/JSC.0000000000000613.

27. MacKenzie B. (2005) 101 Performance Evaluation Tests. London.

28. Mika A., Ł. Oleksy, E. Mikołajczyk (2011) The assessment of the efficacy of the post-exercise muscle recovery modalities using surface electromyography (sEMG). Medical Review of Rzeszow University, 1: 25-39.

29. Minick K., K. Kiesel, L. Burton, A. Taylor, P. Plisky, R. Butler (2010) Interrater reliability of the functional movement screen. J. Strength Cond. Res., 24(2): 479486. DOI: 10.1519/JSC.0b013e3181c09c04.

30. Monroy Antón A.J., B. Rodríguez Rodríguez, D. López Jiménez (2015) Swiss ball training versus stable surface. Arch. Budo, 11: 47-52.

31. Mosler D. (2015) Usability of non-apparatus and quasi apparatus flexibility tests based on self-perception participants in health-related judo training. Arch. Budo Sci. Martial Art. Extreme Sport., 11: 189-197.
32. Mosler D. (2015) Validity and reliability of non-apparatus and quasi apparatus flexibility tests - verification during health-related training based on judo. Arch. Budo Sci. Martial Art. Extreme Sport., 11: 123-133.

33. Okada T., K.C. Huxel, T.W. Nesser (2011) Relationship between core stability, functional movement, and performance. J. Strength Cond. Res., 25(1): 252-261. DOI: 10.1519/JSC.0b013e3181b22b3e.

34. Peacock C., D. Krein, T. Silver (2014) An Acute Bout of Self-Myofascial Release in the Form of Foam Rolling Improves Performance Testing. Int. J. Exerc. Sci., 7(3): 202-211.

35. Silvers-Granelli H., B. Mandelbaum, O. Adeniji, S. Insler, M. Bizzini, R. Pohlig, A. Junge, L. Snyder-Mackler, J. Dvorak (2015) Efficacy of the FIFA 11+ Injury Prevention Program in the Collegiate Male Soccer Player. Am. J. Sport. Med., 2015; September 16, DOI: $10.1177 / 0363546515602009$.

36. Škarabot J., C. Beardsley, I. Štirn (2015) Comparing the effects of self-myofascial release with static stretching on ankle range-of-motion in adolescent athletes. Int. $J$. Sports Phys. Ther, 10(2): 203-212.

37. Stevens D. (2013) Foam rolling as a recovery aid for athletes. J. Aus. Strength Cond., 21(2): 43-51.

38. Sullivan K., D. Silvey, D. Button (2013) Roller Massager Application To The Hamstrings Increases Sit And Reach Range Of Motion Within Five To Ten Seconds Without Performance Impairments, Int. J. Sports Phys. Ther, 8(3): 229-236.

39. Urbaniak M., A. Milańczyk, M. Smoter, A. Zarzycki, D. Mroczek, A. Kawczyński (2015) The effect of deep tissue massage therapy on delayed onset muscle soreness of the lower extremity in karatekas - a preliminary study. J. Combat Sports Martial Arts, 6(1): 7-13. DOI: 10.5604/20815735.1174225.

40. van Mechelen W., J.W. Twisk, G.B. Post, J. Snel, H.C. Kemper (2000) Physical activity of young people: the Amsterdam Longitudinal Growth and Health Study. Med. Sci. Sports Exerc., 32(9): 1610-1616.

41. Weerapong P., P. Hume, G. Kolt (2004) Stretching: mechanisms and benefits for sport performance and injury prevention. Phys. Ther. Rev., 9(4): 189-206. DOI: 10.1179/108331904225007078.

\section{Received 09.03.2017 \\ Accepted 15.05.2017}

(c) University of Physical Education, Warsaw, Poland 